\title{
Bivariate Generalized Exponential Sampling Series and Applications to Seismic Waves
}

\author{
CARlo BARdaro*, Giada Bevignani, Ilaria MANTEllini, AND MARCo SERACini
}

ABSTRACT. In this paper, we introduce the generalized exponential sampling series of bivariate functions and establish some pointwise and uniform convergence results, also in a quantitative form. Moreover, we study the pointwise asymptotic behaviour of the series. One of the basic tools is the Mellin-Taylor formula for bivariate functions, here introduced. A practical application to seismic waves is also outlined.

Keywords: Bivariate generalized exponential sampling series, Mellin-Taylor formulae, moments, magnitude.

2010 Mathematics Subject Classification: 42C15, 46E22, 94A20.

Dedicated to the memory of our close unforgettable Friend and Colleague Professor Domenico Candeloro, who survives in our hearts.

\section{INTRODUCTION}

The classical exponential sampling series of a function $f: \mathbb{R}^{+} \rightarrow \mathbb{C}$, in one dimensional case, represents a tool of relevant interest for optical phenomena, for example the light scattering and Fraunhofer diffraction, see e.g. [17, 11, 18] and [23].

It is defined by

$$
\left(E_{c, T} f\right)(x):=\sum_{k=-\infty}^{\infty} f\left(e^{k / T}\right) \operatorname{lin}_{c / T}\left(e^{-k} x^{T}\right), \quad T>0, x \in \mathbb{R}^{+} .
$$

The $\operatorname{lin}_{c}$-function for $c \in \mathbb{R}, \operatorname{lin}_{c}: \mathbb{R}^{+} \rightarrow \mathbb{R}$, is defined, for $x \in \mathbb{R}^{+} \backslash\{1\}$, by

$$
\operatorname{lin}_{c}(x)=\frac{x^{-c}}{2 \pi i} \frac{x^{\pi i}-x^{-\pi i}}{\log x}=x^{-c} \operatorname{sinc}(\log x)=\frac{x^{-c}}{2 \pi} \int_{-\pi}^{\pi} x^{-i t} d t,
$$

with the continuous extension $\operatorname{lin}_{c}(1):=1$.

Here, the "sinc" function, as usual, is defined by

$$
\operatorname{sinc}(u):=\frac{\sin (\pi u)}{\pi u}, u \neq 0, \operatorname{sinc}(0)=1 .
$$

The exponential sampling theorem for Mellin band-limited functions states that

$$
\left(E_{c, T} f\right)(x)=f(x)
$$

at every point $x$, and the series is absolutely and uniformly convergent on every compact interval of $\mathbb{R}^{+}$. A rigorous treatment of this theorem by a mathematical point of view was given in $[14,3,10]$. This theorem represents a Mellin version of the classical Shannon sampling theorem of Fourier analysis $([20,27])$.

Received: 19 July 2019; Accepted: 11 August 2019; Published Online: 18 August 2019

*Corresponding author: C. Bardaro; carlo.bardaro@unipg.it

DOI: $10.33205 / \mathrm{cma} .594066$ 
Note that, the exponential sampling theorem was framed in the field of the Mellin transform theory, which was first introduced in [22] and then developed in a systematic way in [13].

Now, as it happens for the Fourier band-limited functions, the assumption that $f$ is Mellin band-limited is very restrictive, due to the Mellin-Paley-Wiener theorem (see [4], [5]). Therefore, in a recent paper [7] it was studied a generalization of the one dimensional exponential sampling theorem where the $\operatorname{lin}_{c}$ function is replaced by an arbitrary function $\varphi$ satisfying suitable assumptions in an analogous way as for the generalized sampling series of Fourier analysis (see $[15,16,6,21,2,1])$. In this way, we obtained an approximate reconstruction of a not necessarily Mellin band-limited function $f$.

The aim of this paper is to introduce a multivariate version of the generalized exponential sampling theorem in order to obtain new interesting applications to the study of the seismic waves. In this respect, for a sake of simplicity, we limit ourselves to consider the two dimensional case, being the general case carried on analogously.

Our main theoretical results concern the pointwise and uniform convergence and the study of the pointwise order of approximation through a bivariate asymptotic Voronovskaja formula. Basic tools are a two dimensional Mellin-Taylor formula, established in Section 3 both in the local and global version, and a notion of logarithmic modulus of continuity here introduced as a generalization of one dimensional case (see $[8,9])$.

In Section 6, we give two important examples of bivariate kernel functions satisfying the required assumptions, namely the bivariate Mellin splines and the Mellin-Fejer kernels.

The last section is devoted to the study of the magnitude of an earthquake through the behaviour of the seismic waves.

We wish to dedicate this paper to the memory of our very close friend and colleague Professor Domenico Candeloro who passed away in May. He was a fine mathematician who combined his deep mathematical culture with a great modesty, a trait of his character that makes him an unforgettable person.

\section{PRELIMINARIES}

Let us denote by $\mathbb{N}^{2}, \mathbb{N}_{0}^{2}$ and $\mathbb{Z}^{2}$ the sets of vectors $\mathrm{k}=\left(k_{1}, k_{2}\right)$ with $k_{1}, k_{2}$ positive integers, nonnegative integers and integers respectively and we set $\llbracket \mathrm{k} \|:=k_{1}+k_{2}$. Moreover, by $\mathbb{R}^{2}$ we will denote the two dimensional Euclidean space comprising all vectors $\left(x_{1}, x_{2}\right)$ with $x_{1}, x_{2} \in$ $\mathbb{R}$.

Given $\mathrm{x}=\left(x_{1}, x_{2}\right), \mathrm{y}=\left(y_{1}, y_{2}\right) \in \mathbb{R}^{2}$ we will say that $\mathrm{x}>\mathrm{y}$ if and only if $x_{i}>y_{i}$ for $i=1,2$ and we will denote by $1:=(1,1), 0:=(0,0)$ and by $\mathbb{R}_{+}^{2}$ the space of all vectors $\mathrm{x}>0$.

Given $\mathrm{x}, \mathrm{y} \in \mathbb{R}^{2}$ and $\alpha \in \mathbb{R}$ we put as usual: $\mathrm{x}+\mathrm{y}:=\left(x_{1}+y_{1}, x_{2}+y_{2}\right)$ and $\alpha \mathrm{x}:=\left(\alpha x_{1}, \alpha x_{2}\right)$.

We will employ the following notations: $\mathrm{xy}:=\left(x_{1} y_{1}, x_{2} y_{2}\right), \frac{\mathrm{x}}{\mathrm{y}}:=\left(\frac{x_{1}}{y_{1}}, \frac{x_{2}}{y_{2}}\right)\left(\right.$ for $\left.y_{1}, y_{2} \neq 0\right)$, $[\mathrm{x}]:=\left(\left|x_{1}\right|,\left|x_{2}\right|\right), \alpha^{\mathrm{x}}:=\left(\alpha^{x_{1}}, \alpha^{x_{2}}\right)$ with $\alpha>0$, and $\mathrm{x}^{\mathrm{y}}:=\prod_{i=1}^{2} x_{i}^{y_{i}}, \log (\mathrm{x}):=\left(\log x_{1}, \log x_{2}\right)$ with $x>0$.

We set $\|\mathrm{x}\|:=\sqrt{x_{1}^{2}+x_{2}^{2}}$, and the Euclidean distance $d(\mathrm{x}, \mathrm{y}):=\|\mathrm{x}-\mathrm{y}\|$.

For $\mathrm{w}=\left(w_{1}, w_{2}\right) \in \mathbb{R}_{+}^{2}$, by $\mathrm{w} \rightarrow \infty$ we mean $\underline{w}:=\min \left\{w_{1}, w_{2}\right\} \rightarrow+\infty$.

Let $J$ be an interval, bounded or not. We will denote by $C(J)$ the space of all continuous and bounded functions on $J$, by $C_{c}(J)$ the space of all continuous functions with compact support. Moreover, for $m \in \mathbb{N}$, by $C^{(m)}(J)$ we denote the subspace of $C(J)$ comprising all functions $f$ with the derivatives up to the order $m$ in $C(J)$.

Now, we introduce the following notion of continuity. We will say that a function $f: J \rightarrow \mathbb{C}$ is $\log$-uniformly continuous on $J$ if for every $\varepsilon>0$ there exists $\delta(\varepsilon)>0$ such that $|f(\mathrm{x})-f(\mathrm{y})|<$ 
$\varepsilon$, whenever $\mathrm{x}, \mathrm{y} \in \mathbb{R}_{+}^{2}$ with $\|\log (\mathrm{x})-\log (\mathrm{y})\| \leq \delta(\varepsilon)$. We will denote by $\mathcal{C}(J)$ the space of all log-uniformly continuous and bounded functions on $J$.

Note that for compact intervals $J \subset \mathbb{R}_{+}^{2}$ the notion of log-uniform continuity is equivalent to the classical uniform continuity.

Finally, we will say that a function $f: J \rightarrow \mathbb{C}$ belongs to $C^{(m)}(J)$ locally at a point $\mathrm{x} \in J$ if there is a neighbourhood $I$ of $\mathrm{x}$ such that $f$ is $(m-1)$-times differentiable on $I$ and the derivative $f^{(m)}(\mathrm{x})$ exists.

\section{TWO-DIMENSIONAL MELLIN-TAYLOR FORMULAE}

We begin with the notion of partial derivatives of a function $f: \mathbb{R}_{+}^{2} \rightarrow \mathbb{C}$ in the Mellin frame. The first partial Mellin derivative of $f$ with respect to the variable $x_{i}, i=1,2$ at the point $\mathrm{x}=\left(x_{1}, x_{2}\right)$ is given by

$$
\Theta_{x_{i}} f(\mathrm{x}):=x_{i} \frac{\partial f(\mathrm{x})}{\partial x_{i}} .
$$

For a given $\mathrm{k}=\left(k_{1}, k_{2}\right) \in \mathbb{N}_{0}^{2}$ we define the partial Mellin derivatives of order $r=\llbracket \mathrm{k} \rrbracket=$ $k_{1}+k_{2}$ at the point $\mathrm{x}$ as

$$
\Theta_{x_{1}^{k_{1}} x_{2}^{k_{2}}}^{r} f(\mathrm{x}):=\Theta_{x_{1}}^{k_{1}}\left(\Theta_{x_{2}}^{k_{2}} f\right)(\mathrm{x}) .
$$

We will put $\Theta_{x_{i}}^{1} f(\mathrm{x}):=\Theta_{x_{i}} f(\mathrm{x})$ e $\Theta_{x_{i}}^{0} f(\mathrm{x}):=f(\mathrm{x})$.

Note that for example,

$$
\Theta_{x_{i}}^{2}=\Theta_{x_{i}}\left(\Theta_{x_{i}} f\right)(\mathrm{x})=x_{i} \frac{\partial f(\mathrm{x})}{\partial x_{i}}+x_{i}^{2} \frac{\partial^{2} f(\mathrm{x})}{\partial x_{i}^{2}} .
$$

In order to extend the one-dimensional Mellin-Taylor formulae introduced in [8] to the bivariate case we will use the following notation. For a given $\mathrm{x}=\left(x_{1}, x_{2}\right)$ and $\mathrm{t}=\left(t_{1}, t_{2}\right)$ we set

$$
\begin{aligned}
& \left(\Theta_{x_{1}} \log t_{1}+\Theta_{x_{2}} \log t_{2}\right)^{m} f\left(x_{1}, x_{2}\right) \\
:= & \sum_{k=0}^{m}\left(\begin{array}{c}
m \\
k
\end{array}\right) \Theta_{x_{1}}^{m-k}\left(\Theta_{x_{2}}^{k} f\right)\left(x_{1}, x_{2}\right) \log ^{m-k} t_{1} \log ^{k} t_{2}
\end{aligned}
$$

with $m \in \mathbb{N}$ and $f \in C^{(m)}\left(\mathbb{R}_{+}^{2}\right)$ locally in $\left(x_{1}, x_{2}\right)$.

For example for $m=2$ we obtain

$$
\begin{aligned}
& \left(\Theta_{x_{1}} \log t_{1}+\Theta_{x_{2}} \log t_{2}\right)^{2} f\left(x_{1}, x_{2}\right)= \\
& \Theta_{x_{1}}^{2} f\left(x_{1}, x_{2}\right) \log ^{2} t_{1}+2 \Theta_{x_{1}}\left(\Theta_{x_{2}} f\right)\left(x_{1}, x_{2}\right) \log t_{1} \log t_{2}+\Theta_{x_{2}}^{2} f\left(x_{1}, x_{2}\right) \log ^{2} t_{2} .
\end{aligned}
$$

We have the following proposition.

Proposition 3.1. Let $f: \mathbb{R}_{+}^{2} \rightarrow \mathbb{C}$ be a function in $C^{(m)}\left(\mathbb{R}_{+}^{2}\right)$ with $m \in \mathbb{N}$. Then for $\mathrm{x}=\left(x_{1}, x_{2}\right) \in$ $\mathbb{R}_{+}^{2}$ and $\mathrm{t}=\left(t_{1}, t_{2}\right) \in \mathbb{R}_{+}^{2}$, we have

$$
\begin{aligned}
f\left(t_{1} x_{1}, t_{2} x_{2}\right)= & f\left(x_{1}, x_{2}\right)+\left(\Theta_{x_{1}} \log t_{1}+\Theta_{x_{2}} \log t_{2}\right) f\left(x_{1}, x_{2}\right)+ \\
& \frac{1}{2 !}\left(\Theta_{x_{1}} \log t_{1}+\Theta_{x_{2}} \log t_{2}\right)^{2} f\left(x_{1}, x_{2}\right)+\ldots+ \\
& \frac{1}{(m-1) !}\left(\Theta_{x_{1}} \log t_{1}+\Theta_{x_{2}} \log t_{2}\right)^{m-1} f\left(x_{1}, x_{2}\right)+R_{m}\left(t_{1}, t_{2}\right),
\end{aligned}
$$


with Lagrange remainder

$$
R_{m}\left(t_{1}, t_{2}\right)=\frac{1}{m !}\left(\Theta_{x_{1}} \log t_{1}+\Theta_{x_{2}} \log t_{2}\right)^{m} f(\theta, \eta),
$$

where $(\theta, \eta)$ is a suitable point in the segment $L_{t_{1}, t_{2}}$ with end points $\left(x_{1}, x_{2}\right),\left(t_{1} x_{1}, t_{2} x_{2}\right)$.

Proof. We prove the case $m=2$. For the general case one can apply (3.2).

Let us take the function $F(t)=f\left(t_{1}{ }^{\log t} x_{1}, t_{2}{ }^{\log t} x_{2}\right)$ with $t \in[1, e]$. Applying the one dimensional Mellin-Taylor formula with Lagrange remainder, we obtain

$$
F(t)=F(1)+\Theta F(1) \log t+\frac{\Theta^{2} F(\tilde{t})}{2} \log ^{2} t
$$

with $\tilde{t} \in] 1, e[$. We have

$$
\Theta F(t)=\frac{\partial f}{\partial x_{1}}\left(t_{1}{ }^{\log t} x_{1}, t_{2}{ }^{\log t} x_{2}\right) x_{1} t_{1}{ }^{\log t} \log t_{1}+\frac{\partial f}{\partial x_{2}}\left(t_{1}{ }^{\log t} x_{1}, t_{2}{ }^{\log t} x_{2}\right) x_{2} t_{2}{ }^{\log t} \log t_{2}
$$

and for $t=1$ we obtain

$$
\Theta F(1)=\Theta_{x_{1}} f\left(x_{1}, x_{2}\right) \log t_{1}+\Theta_{x_{2}} f\left(x_{1}, x_{2}\right) \log t_{2}=\left(\Theta_{x_{1}} \log t_{1}+\Theta_{x_{2}} \log t_{2}\right) f\left(x_{1}, x_{2}\right) .
$$

Analogously for $\Theta^{2} F(t)=t F^{\prime}(t)+t^{2} F^{\prime \prime}(t)$, we have

$$
\begin{aligned}
\Theta^{2} F(t)= & \frac{\partial^{2} f}{\partial x_{1}^{2}}\left(t_{1}{ }^{\log t} x_{1}, t_{2}{ }^{\log t} x_{2}\right) x_{1}^{2} t_{1}{ }^{2 \log t} \log ^{2} t_{1}+\frac{\partial^{2} f}{\partial x_{2}^{2}}\left(t_{1}{ }^{\log t} x_{1}, t_{2}{ }^{\log t} x_{2}\right) x_{2}^{2} t_{2}{ }^{2 \log t} \log ^{2} t_{2}+ \\
& 2 \frac{\partial^{2} f}{\partial y \partial x_{1}}\left(t_{1}{ }^{\log t} x_{1}, t_{2}{ }^{\log t} x_{2}\right) x_{1} x_{2}\left(t_{1} t_{2}\right)^{\log t} \log t_{1} \log t_{2}+ \\
& \frac{\partial f}{\partial x_{1}}\left(t_{1}{ }^{\log t} x_{1}, t_{2}{ }^{\log t} x_{2}\right) x_{1} t_{1}{ }^{\log t} \log ^{2} t_{1}+\frac{\partial f}{\partial x_{2}}\left(t_{1}{ }^{\log t} x_{1}, t_{2}{ }^{\log t} x_{2}\right) x_{2} t_{2}{ }^{\log t} \log ^{2} t_{2}
\end{aligned}
$$

and for $t=\tilde{t}$

$$
\begin{aligned}
\frac{\Theta^{2} F(\tilde{t})}{2}= & \frac{1}{2}\left\{\left(\frac{\partial^{2} f}{\partial x_{1}^{2}}(\theta, \eta) \theta^{2}+\frac{\partial f}{\partial x_{1}}(\theta, \eta) \theta\right) \log ^{2} t_{1}+\left(\frac{\partial^{2} f}{\partial x_{2}^{2}}(\theta, \eta) \eta^{2}+\frac{\partial f}{\partial x_{2}}(\theta, \eta) \eta\right) \log ^{2} t_{2}\right. \\
& \left.+2 \frac{\partial^{2} f}{\partial x_{1} \partial x_{2}}(\theta, \eta) \theta \eta \log t_{1} \log t_{2}\right\},
\end{aligned}
$$

with $(\theta, \eta)=\left(t_{1}^{\log \widetilde{t}} x_{1}, t_{2}^{\log \widetilde{t}} x_{2}\right) \in L_{t_{1}, t_{2}}$.

Now, using the definition of the partial Mellin derivative, we have the formulae

$$
\begin{gathered}
\frac{\partial^{2} f}{\partial x_{1}^{2}}(\theta, \eta) \theta^{2}=\left[\Theta_{x_{1}}^{2} f(\theta, \eta)-\Theta_{x_{1}} f(\theta, \eta)\right], \\
\frac{\partial^{2} f}{\partial x_{2}^{2}}(\theta, \eta) \eta^{2}=\left[\Theta_{x_{2}}^{2} f(\theta, \eta)-\Theta_{x_{2}} f(\theta, \eta)\right], \\
\frac{\partial^{2} f}{\partial x_{1} \partial x_{2}}(\theta, \eta) \theta \eta=\Theta_{x_{1}}\left(\Theta_{x_{2}} f\right)(\theta, \eta),
\end{gathered}
$$

then

So the assertion follows.

$$
\frac{\Theta^{2} F(\tilde{t})}{2}=\frac{1}{2}\left(\Theta_{x_{1}} \log t_{1}+\Theta_{x_{2}} \log t_{2}\right)^{2} f(\theta, \eta) .
$$

By Proposition 3.1, we can deduce a local version of the Mellin-Taylor formula, namely a formula with the Peano remainder. It is based on the following proposition 
Proposition 3.2. Under the same assumptions and notations of Proposition 3.1 there holds

$$
\lim _{\left(t_{1}, t_{2}\right) \rightarrow(1,1)} \frac{\left(\Theta_{x_{1}} \log t_{1}+\Theta_{x_{2}} \log t_{2}\right)^{m} f(\theta, \eta)-\left(\Theta_{x_{1}} \log t_{1}+\Theta_{x_{2}} \log t_{2}\right)^{m} f\left(x_{1}, x_{2}\right)}{\left(\log ^{2} t_{1}+\log ^{2} t_{2}\right)^{m / 2}}=0 .
$$

Proof. We consider the case $m=2$, the general case is carried on in a similar way. Setting

$$
I:=\left|\left(\Theta_{x_{1}} \log t_{1}+\Theta_{x_{2}} \log t_{2}\right)^{2} f(\theta, \eta)-\left(\Theta_{x_{1}} \log t_{1}+\Theta_{x_{2}} \log t_{2}\right)^{2} f\left(x_{1}, x_{2}\right)\right|,
$$

we have

$$
\begin{aligned}
& I \leq\left|\Theta_{x_{1}}^{2} f(\theta, \eta)-\Theta_{x_{1}}^{2} f\left(x_{1}, x_{2}\right)\right| \log ^{2} t_{1} \\
+\quad & 2\left|\Theta_{x_{1}}\left(\Theta_{x_{2}} f\right)(\theta, \eta)-\Theta_{x_{1}}\left(\Theta_{x_{2}} f\right)\left(x_{1}, x_{2}\right)\right|\left|\log t_{1} \log t_{2}\right| \\
+\quad & \left|\Theta_{x_{2}}^{2} f(\theta, \eta)-\Theta_{x_{2}}^{2} f\left(x_{1}, x_{2}\right)\right| \log ^{2} t_{2},
\end{aligned}
$$

and hence

$$
\begin{aligned}
\frac{I}{\log ^{2} t_{1}+\log ^{2} t_{2}} \leq & \left|\Theta_{x_{1}}^{2} f(\theta, \eta)-\Theta_{x_{1}}^{2} f\left(x_{1}, x_{2}\right)\right|+\left|\Theta_{x_{1}}\left(\Theta_{x_{2}} f\right)(\theta, \eta)-\Theta_{x_{1}}\left(\Theta_{x_{2}} f\right)\left(x_{1}, x_{2}\right)\right|+ \\
& \left|\Theta_{x_{2}}^{2} f(\theta, \eta)-\Theta_{x_{2}}^{2} f\left(x_{1}, x_{2}\right)\right| .
\end{aligned}
$$

Taking into account that $(\theta, \eta) \in L_{t_{1}, t_{2}}$ the assertion follows from the assumption $f \in C^{(2)}\left(\mathbb{R}_{+}^{2}\right)$.

By Proposition 3.2, we can write the local form of the Mellin-Taylor formula as

$$
\begin{aligned}
f\left(t_{1} x, t_{2} y\right)= & f\left(x_{1}, x_{2}\right)+\left(\Theta_{x_{1}} \log t_{1}+\Theta_{x_{2}} \log t_{2}\right) f\left(x_{1}, x_{2}\right)+ \\
& \frac{1}{2 !}\left(\Theta_{x_{1}} \log t_{1}+\Theta_{x_{2}} \log t_{2}\right)^{2} f\left(x_{1}, x_{2}\right)+\ldots+ \\
& \frac{1}{m !}\left(\Theta_{x_{1}} \log t_{1}+\Theta_{x_{2}} \log t_{2}\right)^{m} f\left(x_{1}, x_{2}\right)+R_{m}\left(t_{1}, t_{2}\right),
\end{aligned}
$$

with the Peano remainder

$$
R_{m}\left(t_{1}, t_{2}\right)=H\left(t_{1}, t_{2}\right)\left(\log ^{2} t_{1}+\log ^{2} t_{2}\right)^{m / 2},
$$

where $H\left(t_{1}, t_{2}\right)$ is a bounded function such that $\lim _{\left(t_{1}, t_{2}\right) \rightarrow(1,1)} H\left(t_{1}, t_{2}\right)=0$.

Remark 3.1. Note that setting $\mathrm{t}=\left(t_{1}, t_{2}\right), \mathrm{x}=\left(x_{1}, x_{2}\right)$ we can write

$$
R_{m}(\mathrm{t})=H(\mathrm{t})\|\log (\mathrm{tx})-\log \mathrm{x}\|^{m} .
$$

Moreover, it is not difficult to see that the local version of the Mellin-Taylor formula can be proved under the more general assumptions $f \in C^{(m)}\left(\mathbb{R}_{+}^{2}\right)$ locally at the point $\mathrm{x}$.

\section{BIVARIATE GENERALIZED SAMPLING OPERATOR}

Let $\varphi: \mathbb{R}_{+}^{2} \rightarrow \mathbb{R}$ be a continuous function such that

( $\varphi .1) \sum_{\mathrm{k} \in \mathbb{Z}^{2}} \varphi\left(e^{-\mathrm{k}} \mathrm{x}\right)=\sum_{\left(k_{1}, k_{2}\right) \in \mathbb{Z}^{2}} \varphi\left(e^{-k_{1}} x_{1}, e^{-k_{2}} x_{2}\right)=1$ for every $\mathrm{x}=\left(x_{1}, x_{2}\right) \in \mathbb{R}_{+}^{2}$;

$(\varphi .2)$ there holds

$$
M_{0}(\varphi):=\sup _{\mathrm{x} \in \mathbb{R}_{+}^{2}} \sum_{\mathrm{k} \in \mathbb{Z}^{2}}\left|\varphi\left(e^{-\mathrm{k}} \mathrm{x}\right)\right|<+\infty ;
$$

$(\varphi .3) \lim _{r \rightarrow+\infty} \sum_{\|\mathrm{k}-\log (\mathrm{x})\| \geq r}\left|\varphi\left(e^{-\mathrm{k}} \mathrm{x}\right)\right|=0$, uniformly with respect to $\mathrm{x}$. 
Let $\Phi$ be the class of all functions $\varphi$ satisfying the above assumptions.

Let $j=\left(j_{1}, j_{2}\right) \in \mathbb{N}_{0}^{2}$ and let $\nu=\rrbracket j \rrbracket$. For $\mathrm{x} \in \mathbb{R}_{+}^{2}$, we define the moments of order $\mathrm{j}$ of $\varphi \in \Phi$ as

$$
\begin{aligned}
& m_{\mathrm{j}}^{\nu}(\varphi, \mathrm{x}):=\sum_{\mathrm{k} \in \mathbb{Z}^{2}} \varphi\left(e^{-\mathrm{k}} \mathrm{x}\right) \log ^{\mathrm{j}}\left(e^{\mathrm{k}} \mathrm{x}^{-1}\right)=\sum_{\mathrm{k} \in \mathbb{Z}^{2}} \varphi\left(e^{-\mathrm{k}} \mathrm{x}\right)(\mathrm{k}-\log (\mathrm{x}))^{\mathrm{j}} \\
= & \sum_{\left(k_{1}, k_{2}\right) \in \mathbb{Z}^{2}} \varphi\left(e^{-k_{1}} x_{1}, e^{-k_{2}} x_{2}\right)\left(k_{1}-\log x_{1}\right)^{j_{1}}\left(k_{2}-\log x_{2}\right)^{j_{2}} .
\end{aligned}
$$

The absolute moments of order $j$ of $\varphi \in \Phi$ are defined as

$$
\begin{aligned}
& M_{\mathrm{j}}^{\nu}(\varphi, \mathrm{x}):=\sum_{\mathrm{k} \in \mathbb{Z}^{2}}\left|\varphi\left(e^{-\mathrm{k}} \mathrm{x}\right)\right|\left[\log \left(e^{\mathrm{k}} \mathrm{x}^{-1}\right)\right]^{\mathrm{j}}=\sum_{\mathrm{k} \in \mathbb{Z}^{2}}\left|\varphi\left(e^{-\mathrm{k}} \mathrm{x}\right)\right|[\mathrm{k}-\log (\mathrm{x})]^{\mathrm{j}} \\
= & \sum_{\left(k_{1}, k_{2}\right) \in \mathbb{Z}^{2}}\left|\varphi\left(e^{-k_{1}} x_{1}, e^{-k_{2}} x_{2}\right)\right|\left|k_{1}-\log x_{1}\right|^{j_{1}}\left|k_{2}-\log x_{2}\right|^{j_{2}} .
\end{aligned}
$$

Finally, we set $M_{\mathrm{j}}^{\nu}(\varphi):=\sup _{\mathrm{x} \in \mathbb{R}_{+}^{2}} M_{\mathrm{j}}^{\nu}(\varphi, \mathrm{x})$.

Let $\varphi \in \Phi$. For any $\mathrm{w}>0, \mathrm{w}=\left(w_{1}, w_{2}\right)$ and $f: \mathbb{R}_{+}^{2} \rightarrow \mathbb{C}$, we define the generalized exponential series as

$$
\left(E_{\mathrm{w}}^{\varphi} f\right)(\mathrm{x}):=\sum_{\mathrm{k} \in \mathbb{Z}^{2}} f\left(e^{\frac{\mathrm{k}}{\mathrm{w}}}\right) \varphi\left(e^{-\mathrm{k}} \mathrm{x}^{\mathrm{w}}\right)=\sum_{\left(k_{1}, k_{2}\right) \in \mathbb{Z}^{2}} f\left(e^{\frac{k_{1}}{w_{1}}}, e^{\frac{k_{2}}{w_{2}}}\right) \varphi\left(e^{-k_{1}} x_{1}^{w_{1}}, e^{-k_{2}} x_{2}^{w_{2}}\right)
$$

for $\mathrm{x}=\left(x_{1}, x_{2}\right) \in \mathbb{R}_{+}^{2}$ and for any function $f \in \operatorname{dom} E_{\mathrm{w}}^{\varphi}$, being $\operatorname{dom} E_{\mathrm{w}}^{\varphi}$ the set of all functions $f$ for which the series is absolutely convergent on every $\mathrm{x}$. Using the conditions of the class $\Phi$, it is easy to see that the above operator is well defined as an absolutely convergent series, for any bounded function $f$. In particular $C\left(\mathbb{R}_{+}^{2}\right) \subset \operatorname{dom} E_{\mathrm{w}}^{\varphi}$, for any w $>0$.

We begin with the following pointwise convergence theorem.

Theorem 4.1. Let $f \in C\left(\mathbb{R}_{+}^{2}\right)$ and $\varphi \in \Phi$. Then

$$
\lim _{\mathrm{w} \rightarrow \infty} \sum_{\mathrm{k} \in \mathbb{Z}^{2}} f\left(e^{\frac{\mathrm{k}}{\mathrm{w}}}\right) \varphi\left(e^{-\mathrm{k}} \mathrm{x}^{\mathrm{w}}\right)=f(\mathrm{x}), \quad \text { for } \quad \mathrm{x} \in \mathbb{R}_{+}^{2} .
$$

Proof. Since $\varphi \in \Phi$, we have

$$
\left|\sum_{\mathrm{k} \in \mathbb{Z}^{2}} f\left(e^{\frac{\mathrm{k}}{\mathrm{w}}}\right) \varphi\left(e^{-\mathrm{k}} \mathrm{x}^{\mathrm{w}}\right)-f(\mathrm{x})\right| \leq \sum_{\mathrm{k} \in \mathbb{Z}^{2}}\left|f\left(e^{\frac{\mathrm{k}}{\mathrm{w}}}\right)-f(\mathrm{x})\right|\left|\varphi\left(e^{-\mathrm{k}} \mathrm{x}^{\mathrm{w}}\right)\right| .
$$

For a fixed $\varepsilon>0$, by the continuity of $f$ at $\mathrm{x}$, there exists $\delta=\delta(\varepsilon)>0$ such that if $\| \log (\mathrm{x})-$ $\log \left(e^{\frac{\mathrm{k}}{\mathrm{w}}}\right)\|=\| \log (\mathrm{x})-\frac{\mathrm{k}}{\mathrm{w}} \|<\delta$, then $\left|f(\mathrm{x})-f\left(e^{\frac{\mathrm{k}}{\mathrm{w}}}\right)\right|<\varepsilon$. We write

$$
\begin{aligned}
& \sum_{\mathrm{k} \in \mathbb{Z}^{2}}\left|f\left(e^{\frac{\mathrm{k}}{\mathrm{w}}}\right)-f(\mathrm{x})\right|\left|\varphi\left(e^{-\mathrm{k}} \mathrm{x}^{\mathrm{w}}\right)\right| \\
= & \left\{\sum_{\left\|\frac{\mathrm{k}}{\mathrm{w}}-\log (\mathrm{x})\right\|<\delta}+\sum_{\left\|\frac{\mathrm{k}}{\mathrm{w}}-\log (\mathrm{x})\right\| \geq \delta}\right\}\left|f\left(e^{\frac{\mathrm{k}}{\mathrm{w}}}\right)-f(\mathrm{x}) \| \varphi\left(e^{-\mathrm{k}} \mathrm{x}^{\mathrm{w}}\right)\right|=: I_{1}+I_{2} .
\end{aligned}
$$


Now by assumption $(\varphi .2)$, we have immediately $I_{1} \leq M_{0}(\varphi) \varepsilon$. As to $I_{2}$ by the boundedness of $f$ and $(\varphi .3)$, taking into account that

$$
\left\|\frac{\mathrm{k}}{\mathrm{W}}-\log (\mathrm{x})\right\| \leq \frac{\left\|\mathrm{k}-\log \left(\mathrm{x}^{\mathrm{w}}\right)\right\|}{\underline{w}}
$$

we have, for sufficiently large $\underline{w}$,

$$
I_{2}=\sum_{\left\|\frac{\mathrm{k}}{\mathrm{w}}-\log (\mathrm{x})\right\| \geq \delta}\left|f\left(e^{\frac{\mathrm{k}}{\mathrm{w}}}\right)-f(\mathrm{x})\left\|\varphi\left(e^{-\mathrm{k}} \mathrm{x}^{\mathrm{w}}\right)\left|\leq 2\|f\|_{\infty} \sum_{\left\|\mathrm{k}-\log \left(\mathrm{x}^{\mathrm{w}}\right)\right\| \geq \delta \underline{w}}\right| \varphi\left(e^{-\mathrm{k}} \mathrm{x}^{\mathrm{w}}\right) \mid<2\right\| f \|_{\infty} \varepsilon,\right.
$$

and so the assertion follows.

Using essentially the same reasoning employed in the previous theorem, we can prove the following uniform convergence result.

Theorem 4.2. Let $f \in \mathcal{C}\left(\mathbb{R}_{+}^{2}\right)$ and $\varphi \in \Phi$, then

$$
\lim _{\mathrm{w} \rightarrow \infty}\left\|\sum_{\mathrm{k} \in \mathbb{Z}^{2}} f\left(e^{\frac{\mathrm{k}}{\mathrm{w}}}\right) \varphi\left(e^{-\mathrm{k}} \mathrm{x}^{\mathrm{w}}\right)-f(\mathrm{x})\right\|_{\infty}=0 .
$$

\section{ESTIMATION OF THE ERROR OF APPROXIMATION}

We premise the following notion. The logarithmic modulus of continuity of $f \in \mathcal{C}\left(\mathbb{R}_{+}^{2}\right)$ is defined, for $\delta>0$, by

$$
\omega(f, \delta):=\sup \left\{|f(\mathrm{x})-f(\mathrm{y})|: \mathrm{x}, \mathrm{y} \in \mathbb{R}_{+}^{2}, \quad\|\log (\mathrm{x})-\log (\mathrm{y})\| \leq \delta\right\} .
$$

This modulus satisfies all the properties of the one dimensional logarithmic modulus of continuity (see [9]). In particular, it is a monotone increasing function of $\delta>0$ and the following inequality holds, for $\lambda>0$

$$
\omega(f, \lambda \delta) \leq(1+\lambda) \omega(f, \delta) .
$$

We have the following theorem.

Theorem 5.3. If $f \in \mathcal{C}\left(\mathbb{R}_{+}^{2}\right), \varphi \in \Phi$, and

$$
D:=\sup _{\mathrm{x} \in \mathbb{R}_{+}^{2}} \sum_{\mathrm{k} \in \mathbb{Z}^{2}}\left|\varphi\left(e^{-\mathrm{k}} \mathrm{x}\right)\right|\|\mathrm{k}-\log \mathrm{x}\|<+\infty,
$$

then for $\mathrm{w}>0$ and $\delta>0$, we have

$$
\left|\sum_{\mathrm{k} \in \mathbb{Z}^{2}} f\left(e^{\frac{\underline{k}}{\underline{w}}}\right) \varphi\left(e^{-\mathrm{k}} \mathrm{x}^{\mathrm{w}}\right)-f(\mathrm{x})\right| \leq M_{0}(\varphi) \omega(f, \delta)+D \frac{\omega(f, \delta)}{\delta \underline{w}} .
$$

Proof. Using that $\varphi \in \Phi,(5.6)$ and the inequality

we have

$$
\left\|\frac{\mathrm{k}}{\mathrm{W}}-\log (\mathrm{x})\right\| \leq \frac{\left\|\mathrm{k}-\log \left(\mathrm{x}^{\mathrm{w}}\right)\right\|}{\underline{w}},
$$

$$
\begin{aligned}
& \left|E_{\mathrm{w}}^{\varphi} f(\mathrm{x})-f(\mathrm{x})\right| \leq \omega(f, \delta)\left(\sum_{\mathrm{k} \in \mathbb{Z}^{2}}\left|\varphi\left(e^{-\mathrm{k}} \mathrm{x}^{\mathrm{w}}\right)\right|+\sum_{\mathrm{k} \in \mathbb{Z}^{2}}\left|\varphi\left(e^{-\mathrm{k}} \mathrm{x}^{\mathrm{w}}\right)\right| \frac{\left\|\mathrm{k}-\log \left(\mathrm{x}^{\mathrm{w}}\right)\right\|}{\delta \underline{w}}\right) \\
& \leq \omega(f, \delta) M_{0}(\varphi)+\frac{\omega(f, \delta)}{\delta \underline{w}} D
\end{aligned}
$$

and so the assertion follows. 
As a corollary we can prove

Corollary 5.1. Under the assumptions of Teorem 5.3 there holds

$$
\left|\sum_{\mathrm{k} \in \mathbb{Z}^{2}} f\left(e^{\frac{\mathrm{k}}{w}}\right) \varphi\left(e^{-\mathrm{k}} \mathrm{x}^{\mathrm{w}}\right)-f(\mathrm{x})\right| \leq C(\varphi) \omega\left(f, \frac{1}{\underline{w}}\right) .
$$

Proof. Applying Theorem 5.3 with $\delta=\frac{1}{\underline{w}}$, we obtain

$$
\left|E_{\mathrm{w}}^{\varphi} f(\mathrm{x})-f(\mathrm{x})\right| \leq \omega\left(f, \frac{1}{\underline{w}}\right) M_{0}(\varphi)+\omega\left(f, \frac{1}{\underline{w}}\right) D .
$$

Setting $C(\varphi)=M_{0}(\varphi)+D$ we have the assertion.

Now, we obtain estimations of the order of approximation under some local regularity assumptions on the function $f$. In order to do that we will need further assumptions on the kernel function $\varphi$, i.e., there exists $\ell \in \mathbb{N}$ such that for every $j \in \mathbb{N}_{0}^{2},\|j\| \leq \ell$

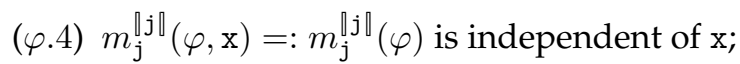

$(\varphi .5) M_{\mathrm{j}}^{\square j \rrbracket}(\varphi)<+\infty$ and

$$
\lim _{r \rightarrow+\infty} \sum_{\|\mathrm{k}-\log (\mathrm{x})\|>r}\left|\varphi\left(e^{-\mathrm{k}} \mathrm{x}\right)\right|\|(\mathrm{k}-\log (\mathrm{x}))\|^{\ell}=0
$$

uniformly with respect to $\mathrm{x}$.

Remark 5.2. Since for any $\mathrm{j}=\left(j_{1}, j_{2}\right) \in \mathbb{N}_{0}^{2}$ and any vector $\mathrm{v}=\left(v_{1}, v_{2}\right) \in \mathbb{R}_{+}^{2}$ we have $[\mathrm{v}]^{\mathrm{j}} \leq$ $\|\mathrm{v}\|^{\mathrm{j}}$, we deduce immediately that assumption $(\varphi .5)$ implies that

$$
\lim _{r \rightarrow+\infty} \sum_{\|\mathrm{k}-\log (\mathrm{x})\|>r}\left|\varphi\left(e^{-\mathrm{k}} \mathrm{x}\right)\right|[\mathrm{k}-\log (\mathrm{x})]^{\rrbracket j \rrbracket}=0,
$$

uniformly with respect to $x$, for every $j$ with $\llbracket j \rrbracket \leq \ell$.

We denote by $\Phi_{\ell}$ the set of functions $\varphi$ satisfying conditions $(\varphi .1),(\varphi .4),(\varphi .5)$.

We have the following result, in which we assume $\ell=2$.

Theorem 5.4. Let $f: \mathbb{R}_{+}^{2} \rightarrow \mathbb{C}$ be a function such that $f \in C^{(2)}\left(\mathbb{R}_{+}^{2}\right)$ locally at the point $\mathrm{x}=$ $\left(x_{1}, x_{2}\right) \in \mathbb{R}_{+}^{2}$. If $\varphi \in \Phi_{2}$, then for $\mathrm{w}=\left(w_{1}, w_{2}\right)>0$,

$$
\left(E_{\left(w_{1}, w_{2}\right)}^{\varphi} f\right)\left(x_{1}, x_{2}\right)-f\left(x_{1}, x_{2}\right)=\sum_{\nu=1}^{2} \sum_{\llbracket \mathrm{h} \rrbracket=\nu}\left(\frac{\Theta^{\nu} f\left(x_{1}, x_{2}\right.}{\nu !} \frac{m_{h}^{\nu}(\varphi)}{\mathrm{w}^{\mathrm{h}}}\right)+o\left(\underline{w}^{-2}\right) .
$$


Proof. Since $\varphi \in \Phi_{2}$, applying the Mellin-Taylor formula of the second order with local remainder, we can write

$$
\begin{aligned}
& \left(E_{\left(w_{1}, w_{2}\right)}^{\varphi} f\right)\left(x_{1}, x_{2}\right)-f\left(x_{1}, x_{2}\right)=\sum_{\left(k_{1}, k_{2}\right) \in \mathbb{Z}^{2}} \varphi\left(e^{-k_{1}} x_{1}^{w_{1}}, e^{-k_{2}} x_{2}^{w_{2}}\right)\left(\left(\Theta_{x_{1}} \log \left(\frac{e^{\frac{k_{1}}{w_{1}}}}{x_{1}}\right)+\right.\right. \\
& \left.\Theta_{x_{2}} \log \left(\frac{e^{\frac{k_{2}}{w_{2}}}}{x_{2}}\right)\right) f\left(x_{1}, x_{2}\right)+\frac{1}{2}\left(\Theta_{x_{1}} \log \left(\frac{e^{\frac{k_{1}}{w_{1}}}}{x_{1}}\right)+\Theta_{x_{2}} \log \left(\frac{e^{\frac{k_{2}}{w_{2}}}}{x_{2}}\right)\right)^{2} f\left(x_{1}, x_{2}\right)+ \\
& \left.H\left(\frac{e^{\frac{k_{1}}{w_{1}}}}{x_{1}}, \frac{e^{\frac{k_{2}}{w_{2}}}}{x_{2}}\right)\left(\log ^{2}\left(\frac{e^{\frac{k_{1}}{w_{1}}}}{x_{1}}\right)+\log ^{2}\left(\frac{e^{\frac{k_{2}}{w_{2}}}}{x_{2}}\right)\right)\right) \\
& =\frac{1}{w_{1}} \Theta_{x_{1}} f\left(x_{1}, x_{2}\right) m_{(1,0)}^{1}(\varphi)+\frac{1}{w_{2}} \Theta_{x_{2}} f\left(x_{1}, x_{2}\right) m_{(0,1)}^{1}(\varphi)+\frac{1}{2} \frac{1}{w_{1}^{2}} \Theta_{x_{1}}^{2} f(x, y) m_{(2,0)}^{2}(\varphi)+ \\
& \frac{1}{2} \frac{1}{w_{2}^{2}} \Theta_{x_{2}}^{2} f\left(x_{1}, x_{2}\right) m_{(0,2)}^{2}(\varphi)+\frac{1}{w_{1}} \frac{1}{w_{2}} \Theta_{x_{1}}\left(\Theta_{x_{2}} f\right)\left(x_{1}, x_{2}\right) m_{(1,1)}^{2}(\varphi)+ \\
& \sum_{\left(k_{1}, k_{2}\right) \in \mathbb{Z}^{2}} \varphi\left(e^{-k_{1}} x_{1}^{w_{1}}, e^{-k_{2}} x_{2}^{w_{2}}\right) H\left(\frac{e^{\frac{k_{1}}{w_{1}}}}{x_{1}}, \frac{e^{\frac{k_{2}}{w_{2}}}}{x_{2}}\right)\left(\log ^{2}\left(\frac{e^{\frac{k_{1}}{w_{1}}}}{x_{1}}\right)+\log ^{2}\left(\frac{e^{\frac{k_{2}}{w_{2}}}}{x_{2}}\right)\right) \\
& =\sum_{\nu=1}^{2} \sum_{|h|=\nu}\left(\frac{\Theta^{\nu} f\left(x_{1}, x_{2}\right)}{\nu !} \frac{m_{h}^{\nu}(\varphi)}{w^{\mathrm{h}}}\right)+ \\
& \sum_{\left(k_{1}, k_{2}\right) \in \mathbb{Z}^{2}} \varphi\left(e^{-k_{1}} x_{1}^{w_{1}}, e^{-k_{2}} x_{2}^{w_{2}}\right) H\left(\frac{e^{\frac{k_{1}}{w_{1}}}}{x_{1}}, \frac{e^{\frac{k_{2}}{w_{2}}}}{x_{2}}\right)\left(\log ^{2}\left(\frac{e^{\frac{k_{1}}{w_{1}}}}{x_{1}}\right)+\log ^{2}\left(\frac{e^{\frac{k_{2}}{w_{2}}}}{x_{2}}\right)\right) .
\end{aligned}
$$

Here, $H\left(t_{1}, t_{2}\right)$ tends to zero, for $\left(t_{1}, t_{2}\right) \rightarrow(1,1)$. Thus, for a given, $\varepsilon>0$ there is $\delta>0$ such that

$$
H\left(\frac{e^{k_{1} / w_{1}}}{x_{1}}, \frac{e^{k_{2} / w_{2}}}{x_{2}}\right)<\varepsilon
$$

whenever $\left\|\log \left(e^{\mathrm{k} / \mathrm{w}}\right)-\log (\mathrm{x})\right\|<\delta$.

Setting

$$
R:=\sum_{\left(k_{1}, k_{2}\right) \in \mathbb{Z}^{2}} \varphi\left(e^{-k_{1}} x_{1}^{w_{1}}, e^{-k_{2}} x_{2}^{w_{2}}\right) H\left(\frac{e^{\frac{k_{1}}{w_{1}}}}{x_{1}}, \frac{e^{\frac{k_{2}}{w_{2}}}}{x_{2}}\right)\left(\log ^{2}\left(\frac{e^{\frac{k_{1}}{w_{1}}}}{x_{1}}\right)+\log ^{2}\left(\frac{e^{\frac{k_{2}}{w_{2}}}}{x_{2}}\right)\right),
$$

taking into account that

$$
\left\|\frac{\mathrm{k}}{\mathrm{W}}-\log (\mathrm{x})\right\| \leq \frac{\left\|\mathrm{k}-\log \left(\mathrm{x}^{\mathrm{w}}\right)\right\|}{\underline{w}}
$$

as in Theorem 4.1, we obtain

$$
\begin{aligned}
& \underline{w}^{2}|R| \leq \sum_{\left(k_{1}, k_{2}\right) \in \mathbb{Z}^{2}}\left|\varphi\left(e^{-k_{1}} x_{1}^{w_{1}}, e^{-k_{2}} x_{2}^{w_{2}}\right)\right|\left|H\left(\frac{e^{\frac{k_{1}}{w_{1}}}}{x_{1}}, \frac{e^{\frac{k_{2}}{w_{2}}}}{x_{2}}\right)\right|\left\|\mathrm{k}-\log \left(\mathrm{x}^{\mathrm{w}}\right)\right\|^{2} \\
\leq & \left\{\sum_{\|\underline{\underline{k}}-\log (\mathrm{x})\|<\delta}+\sum_{\left\|\mathrm{k}-\log \left(\mathrm{x}^{\mathrm{w}}\right)\right\| \geq \delta \underline{w}}\right\}\left|\varphi\left(e^{-k_{1}} x_{1}^{w_{1}}, e^{-k_{2}} x_{2}^{w_{2}}\right)\right|\left|H\left(\frac{e^{\frac{k_{1}}{w_{1}}}}{x_{1}}, \frac{e^{\frac{k_{2}}{w_{2}}}}{x_{2}}\right)\right|\left\|\mathrm{k}-\log \left(\mathrm{x}^{\mathrm{w}}\right)\right\|^{2} \\
=: & S_{1}+S_{2} .
\end{aligned}
$$


As to $S_{1}$, denoting $M_{2}(\varphi):=\max _{\llbracket j \rrbracket=2} M_{j}^{2}(\varphi)$, we have easily $S_{1} \leq 3 M_{2}(\varphi) \varepsilon$, while for $S_{2}$, by $(\varphi .5)$ and the boundedness of $H$, we obtain $S_{2} \leq\|H\|_{\infty} \varepsilon$. Thus the proof is complete.

\section{SOME EXAMPLES}

In [7] the one-dimensional generalized exponential sampling series was introduced, in which the generating (one-dimensional) kernel $\phi: \mathbb{R}^{+} \rightarrow \mathbb{R}$ satisfies the assumptions:

( $\phi .1) \sum_{k \in \mathbb{Z}} \phi\left(e^{-k} x\right)=1$ for every $x \in \mathbb{R}^{+}$,

$(\phi .2) \sup _{x \in \mathbb{R}^{+}} \sum_{k \in \mathbb{Z}}\left|\phi\left(e^{-k} x\right)\right|<+\infty$;

( $\phi .3) \lim _{r \rightarrow+\infty} \sum_{|k-\log (x)|>r}\left|\phi\left(e^{-k} x\right)\right|=0$, uniformly with respect to $x$.

We will denote by $\Psi$ the set comprising all functions $\phi: \mathbb{R}^{+} \rightarrow \mathbb{R}$ satisfying $(\phi .1),(\phi .2)$ and $(\phi .3)$. Using a product of two such functions, we can construct examples of two-dimensional kernel $\varphi \in \Phi$. In this respect we have the following proposition.

Proposition 6.3. If $\phi_{1}, \phi_{2} \in \Psi$ are bounded, then $\Gamma\left(x_{1}, x_{2}\right):=\phi_{1}\left(x_{1}\right) \phi_{2}\left(x_{2}\right) \in \Phi$.

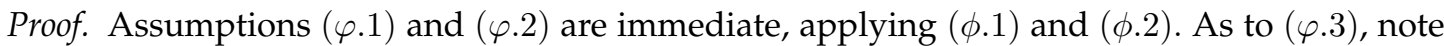
that if $\left\|\left(k_{1}-\log \left(x_{1}\right), k_{2}-\log \left(x_{2}\right)\right)\right\|>r$, then $r<\left\|\left(k_{1}-\log \left(x_{1}\right), k_{2}-\log \left(x_{2}\right)\right)\right\| \leq\left|k_{1}-\log \left(x_{1}\right)\right|+$ $\left|k_{2}-\log \left(x_{2}\right)\right|$. Therefore,

$$
\begin{aligned}
& \lim _{r \rightarrow+\infty} \sum_{||\left(k_{1}-\log \left(x_{1}\right), k_{2}-\log (y)\right) \|>r}\left|\varphi_{1}\left(e^{-k_{1}} x\right) \varphi_{2}\left(e^{-k_{2}} x_{2}\right)\right| \\
& \leq \lim _{r \rightarrow+\infty} \sum_{\left|k_{1}-\log \left(x_{1}\right)\right|+\left|k_{2}-\log \left(x_{2}\right)\right|>r}\left|\varphi_{1}\left(e^{-k_{1}} x\right) \varphi_{2}\left(e^{-k_{2}} x_{2}\right)\right| .
\end{aligned}
$$

For a fixed $\mathrm{x}=\left(x_{1}, x_{2}\right) \in \mathbb{R}_{+}^{2}$ and $r>0$, we set

$$
\begin{gathered}
A:=\left\{\left(k_{1}, k_{2}\right):\left|k_{1}-\log \left(x_{1}\right)\right|+\left|k_{2}-\log \left(x_{2}\right)\right|>r\right\} ; \\
B_{1}:=\left\{\left(k_{1}, k_{2}\right):\left|k_{1}-\log \left(x_{1}\right)\right|>\frac{r}{2}\right\}, \quad B_{2}:=\left\{\left(k_{1}, k_{2}\right):\left|k_{2}-\log \left(x_{2}\right)\right|>\frac{r}{2}\right\}
\end{gathered}
$$

and $B:=B_{1} \cup B_{2}$. Since $A \subset B$, we have

$$
\begin{aligned}
& \lim _{r \rightarrow+\infty} \sum_{\left(k_{1}, k_{2}\right) \in A}\left|\varphi_{1}\left(e^{-k_{1}} x_{1}\right) \varphi_{2}\left(e^{-k_{2}} x_{2}\right)\right| \leq \lim _{r \rightarrow+\infty} \sum_{\left(k_{1}, k_{2}\right) \in B}\left|\varphi_{1}\left(e^{-k_{1}} x_{1}\right) \varphi_{2}\left(e^{-k_{2}} x_{2}\right)\right| \\
& \leq \lim _{r \rightarrow+\infty} \sum_{\left(k_{1}, k_{2}\right) \in B_{1}}\left|\varphi_{1}\left(e^{-k_{1}} x_{1}\right) \varphi_{2}\left(e^{-k_{2}} x_{2}\right)\right|+\lim _{r \rightarrow+\infty} \sum_{\left(k_{1}, k_{2}\right) \in B_{2}}\left|\varphi_{1}\left(e^{-k_{1}} x_{1}\right) \varphi_{2}\left(e^{-k_{2}} x_{2}\right)\right| .
\end{aligned}
$$

By the boundedness of the functions $\phi_{1}$ and $\phi_{2}$ and by $(\phi .3)$, we obtain easily $(\varphi .3)$.

Making use of Proposition 6.3, we costruct some box-type kernel, using the product of two classical one-dimensional kernels.

Example 6.1. Denoting by $r_{+}$the positive part of a number $r \in \mathbb{R}$, for $n \in \mathbb{N}$, we define the (one-dimensional) Mellin spline of order $n$, as (see [7, 10]

$$
B_{n}(x):=\frac{1}{(n-1) !} \sum_{j=0}^{n}(-1)^{j}\left(\begin{array}{l}
n \\
j
\end{array}\right)\left(\frac{n}{2}+\log x-j\right)_{+}^{n-1}, \quad\left(x \in \mathbb{R}^{+}\right) .
$$


These kernel functions are the Mellin version of the classical central $B$-splines see [25]. The functions $B_{n}$ are compactly supported, and satisfy all the assumptions of the class $\Psi$ (see [7]). Using these functions we can define, for $n, m \in \mathbb{N}$,

$$
B_{n, m}\left(x_{1}, x_{2}\right):=B_{n}\left(x_{1}\right) B_{m}\left(x_{2}\right) .
$$

By Proposition 6.3, the kernel $B_{n, m} \in \Phi$. In particular, for $n=m=2$, we obtain

$$
B_{2,2}\left(x_{1}, x_{2}\right)= \begin{cases}\left(1+\log x_{1}\right)\left(1+\log x_{2}\right), & e^{-1}<x_{1}, x_{2}<1 \\ \left(1-\log x_{1}\right)\left(1+\log x_{2}\right), & 1<x_{1}<e, e^{-1}<x_{2}<1 \\ \left(1+\log x_{1}\right)\left(1-\log x_{2}\right), & e^{-1}<x_{1}<1,1<x_{2}<e \\ \left(1-\log x_{1}\right)\left(1-\log x_{2}\right), & 1<x_{1}, x_{2}<e \\ 0, & \text { otherwise. }\end{cases}
$$

Example 6.2. Another interesting example, is given by the product of two one-dimensional Mellin-Fejer kernels, which are defined for any $\rho>0, c \in \mathbb{R}$ and $x \in \mathbb{R}^{+}$by $($see $[7,10])$

$$
F_{\rho}^{c}(x):=\left\{\begin{array}{ll}
\frac{x^{-c}}{2 \pi} \rho \operatorname{sinc}^{2}\left(\frac{\rho}{\pi} \log \sqrt{x}\right), & x \neq 1 \\
\frac{\rho}{2 \pi}, & x=1
\end{array} .\right.
$$

We have $F_{\rho}^{c} \in \Psi$ and using again Proposition 6.3, we see that the kernel

$$
F_{\rho_{1}, \rho_{2}}^{c_{1}, c_{2}}\left(x_{1}, x_{2}\right)=F_{\rho_{1}}^{c_{1}}\left(x_{1}\right) F_{\rho_{2}}^{c_{2}}\left(x_{2}\right) \quad\left(x_{1}, x_{2}\right) \in \mathbb{R}_{+}^{2},
$$

with $\rho_{1}, \rho_{2}>0, c_{1}, c_{2} \in \mathbb{R}$, belongs to the class $\Phi$.

Remark 6.3. As remarked in [7], the one-dimensional Mellin-Fejer kernel does not satisfy the (one-dimensional) moment condition

$$
\widetilde{M}_{1}(\phi):=\sup _{x \in \mathbb{R}^{+}} \sum_{k=-\infty}^{\infty}\left|\varphi\left(e^{-k} x\right)\right||k-\log x|<+\infty .
$$

Therefore, in $[7,10]$ a suitable modification of the kernel was studied, introducing the so-called Mellin-Jackson kernels, which satisfy the above condition. Thus, one can obtain other interesting examples, by considering the product of two Mellin-Jackson kernels.

\section{SEISMIC WAVES AND EXPONENTIAL SAMPLING}

Due to their nature and their way of propagation, seismic waves can be modeled using exponential functions. Seismic waves, due to the continuous and natural movements of the terrestrial plates, originate from a point at a certain depth into the ground, this point called hypocenter, and develop for several kilometers, being attenuated thanks to the elastic properties of the medium they cross. The projection of the hypocenter on the heart surface is called epicenter and it is the point of maximum amplitude of the seismic wave. For a given direction $\theta \in[-\pi, \pi]$ in the horizontal plane, the definition of amplitude $A(R, \theta)$ of a seismic wave, used in this work, is the variation, measured in $\mathrm{mm}$, registered by a standard Wood-Anderson seismograph at a certain distance $R$ from the epicenter. Connected with the definition of $A(R, \theta)$ is the formalization of the Local Magnitude $\operatorname{LM}(R, \theta)$ [12], needed to measure the intensity of an earthquake according to the Richter scale $[19,24]$ :

$$
L M(R, \theta):=\log _{10} A(R, \theta)-\log _{10} A_{0}(R, \theta),
$$


where $\log _{10} A_{0}(R, \theta)$ is a calibration function such that, for $R=100 \mathrm{Km}$ and in whatever direction $\theta, \log _{10} A_{0}(100, \theta)=3$. We assume the model to be symmetric with respect to the epicenter that is located in the center of the axis $((0,0)$ coordinates).

The model derived in [12], based on the dataset provided by ISNet (Irpinia Seismic Network), has been used to test how the mathematical theory approximates real data. ISNet is a network of 27 stations located in the South of Italy, along the Apennines chain [26]. In the ISNet dataset the epicenter distance $R$ has been substituted with the hypocenter distance, committing an error of less than $1 \%$, approximation possible thanks to the reduced depth $(<20 \mathrm{Km})$ of the hypocenter in the ISNet data. To approximate the real data the following model has been used:

$$
L M(R, \theta)=\log _{10} A(R, \theta)-\alpha \log _{10} R-k R-\beta
$$

where $\alpha=-1.79, \beta=0.58, k=0$. The values of the parameters have been achieved considering a minimization criteria, according to the elastic structural parameters characterizing the area monitored by the ISNet network. In the light of the previous considerations, the model assumes the form (see [12] for all the details):

$$
L M(R, \theta)=\log _{10} A(R, \theta)+1.79 \log _{10} R-0.58
$$

from which, in case of invertibility, we can write the inverse formulation:

$$
A(R, \theta, L M)=10^{\left(L M(R, \theta)-1.79 \log _{10} R+0.58\right),}
$$

or equivalently in cartesian coordinates:

$$
A\left(R_{1}, R_{2}, L M\right)=10\left(L M\left(R_{1}, R_{2}\right)-1.79 \log _{10} \arctan \left(R_{1} / R_{2}\right)+0.58\right),
$$

where $R_{1}$ and $R_{2}$ are, respectively, the horizontal and vertical cartesian axis such that $R=$ $\arctan \left(R_{1} / R_{2}\right), R_{1}=R \cos (\theta), R_{2}=R \sin (\theta)$. Fixed a value for $L M\left(R_{1}, R_{2}\right)$, it is possible to calculate $A\left(R_{1}, R_{2}\right)$ and to approximate it with an exponential sampling operator, defined as:

$$
\left(E_{\left(w_{1}, w_{2}\right)}^{\varphi} A\right)\left(R_{1}, R_{2}\right)=\sum_{\left(k_{1}, k_{2}\right) \in \mathbb{Z}^{2}} A\left(e^{\frac{k_{1}}{w_{1}}}, e^{\frac{k_{2}}{w_{2}}}\right) \varphi\left(e^{-k_{1}} R_{1}{ }^{w_{1}}, e^{-k_{2}} R_{2}{ }^{w_{2}}\right),
$$

with $R_{1}, R_{2}$ integers.

Finally, a quantification of the reconstruction absolute mean error $A M E$ has been provided introducing the following error estimator

$$
A M E:=\frac{1}{N_{1} N_{2}} \sum_{R_{1}=0}^{N_{1}} \sum_{R_{2}=0}^{N_{2}}\left|\left(E_{\left(w_{1}, w_{2}\right)}^{\varphi} A\right)\left(R_{1}, R_{2}\right)-A\left(R_{1}, R_{2}\right)\right| .
$$

In the previous expression $N_{1}$ and $N_{2}$ are the number of points in the two main cartesian axis directions, $N_{1} \times N_{2}$ being the total number of the samples in the grid and $R_{1} \in\left[0, N_{1}\right]$, $R_{2} \in\left[0, N_{2}\right]$.

Chosen $L M=2.7$ in the ISNet dataset, we achieve, for bivariate Mellin-Fejer kernels, the numerical values shown in table 1, where only a single row of the approximating matrix is reported for practical reasons. Other rows of the same matrix exhibit the same trend. Using Mellin Splines kernels, we achieve better approximation results (see table 2). In any case, increasing $w_{1}, w_{2}, N_{1}, N_{2}$ we observe as $A M E$ decreases, whatever kernel being used in the approximation formula. 


\begin{tabular}{rrrr}
\hline $\mathrm{A}$ & $\mathrm{N}=15, \mathrm{w}=5$ & $\mathrm{~N}=30, \mathrm{w}=10$ & $\mathrm{~N}=60, \mathrm{w}=20$ \\
\hline 918.4869 & 2016.2750 & 1420.2633 & 1182.1876 \\
907.7103 & 1545.3763 & 1213.2350 & 1064.3751 \\
890.3316 & 1319.0114 & 1090.6358 & 990.5768 \\
867.1510 & 1169.1624 & 998.5332 & 932.5291 \\
839.1579 & 1046.1715 & 926.9082 & 880.6653 \\
807.4335 & 945.1902 & 861.7557 & 832.4209 \\
773.0601 & 862.8483 & 803.7728 & 786.1427 \\
737.0491 & 794.8578 & 752.2213 & 741.7739 \\
700.2923 & 737.3727 & 704.8035 & 698.9664 \\
663.5358 & 687.5171 & 660.3791 & 658.5373 \\
627.3745 & 643.3295 & 618.9350 & 619.9301 \\
592.2586 & 603.5319 & 580.7086 & 583.3547 \\
558.5099 & 567.3041 & 545.7103 & 549.1905 \\
526.3414 & 534.1130 & 513.6886 & 517.2242 \\
495.8780 & 503.5975 & 484.2704 & 487.1994 \\
467.1756 & 475.4960 & 457.0936 & 459.1393 \\
440.2385 & 449.6028 & 431.8738 & 433.1069 \\
415.0331 & 425.7436 & 408.4140 & 408.9969 \\
391.4999 & 403.7617 & 386.5841 & 386.5923 \\
369.5621 & 383.5117 & 366.2922 & 365.7069 \\
\hline
\end{tabular}

TABLE 1. In the first column of the table the values of the $15^{\text {th }}$ row of the real data for $L M=2.7$. In the following columns, from left to right, the reconstructed data with bivariate Mellin-Fejer kernels with $N=N_{1}=N_{2}, w=$ $w_{1}=w_{2}$ respectively equal to $15,30,60$ and $5,10,20$.

\section{ACKNOWLEDGMENTS}

We wish to thank the referees for their useful suggestions which improved the presentation of the paper.

Carlo Bardaro, Ilaria Mantellini and Marco Seracini have been partially supported by the "Gruppo Nazionale per l'Analisi Matematica e Applicazioni (GNAMPA)" of the "Instituto di Alta Matematica (INDAM)" . Carlo Bardaro and Ilaria Mantellini have been partially supported also by the projects "Ricerca di Base 2017 of University of Perugia (title: Misura, Integrazione, Approssimazione e loro Applicazioni)" and "Progetto Fondazione Cassa di Risparmio cod. nr. 2018.0419.021 (title: Metodi e Processi di Intelligenza artificiale per lo sviluppo di una banca di immagini mediche per fini diagnostici (B.I.M.))". 


\begin{tabular}{rrrr}
\hline $\mathrm{A}$ & $\mathrm{N}=15, \mathrm{w}=5$ & $\mathrm{~N}=30, \mathrm{w}=10$ & $\mathrm{~N}=60, \mathrm{w}=20$ \\
\hline 918.4869 & 919.7995 & 919.1974 & 918.8313 \\
907.7103 & 908.9871 & 908.3652 & 908.0278 \\
890.3316 & 891.5131 & 890.9341 & 890.6223 \\
867.1510 & 867.3503 & 867.5869 & 867.3689 \\
839.1579 & 838.9015 & 839.5563 & 839.3507 \\
807.4335 & 806.7726 & 807.6950 & 807.5622 \\
773.0601 & 772.5943 & 772.9821 & 773.1665 \\
737.0491 & 736.8876 & 737.0027 & 737.0632 \\
700.2923 & 699.0600 & 700.4001 & 700.3419 \\
663.5358 & 663.8368 & 663.6755 & 663.5998 \\
627.3745 & 626.2857 & 627.4080 & 627.3887 \\
592.2586 & 592.0042 & 592.1529 & 592.2225 \\
558.5099 & 557.9946 & 558.3477 & 558.4872 \\
526.3414 & 525.8575 & 526.2417 & 526.3176 \\
495.8780 & 495.8853 & 495.8670 & 495.8693 \\
467.1756 & 467.2693 & 467.1553 & 467.1692 \\
440.2385 & 440.3887 & 440.2862 & 440.2415 \\
415.0331 & 415.0450 & 415.0472 & 415.0398 \\
391.4999 & 392.0046 & 391.6462 & 391.5060 \\
369.5621 & 370.4046 & 369.5769 & 369.5696 \\
\hline
\end{tabular}

TABLE 2. In the first column of the table the values of the $15^{\text {th }}$ row of the real data for $L M=2.7$. In the following columns, from left to right, the reconstructed data with the bivariate Mellin-Splines $B_{2,2}$ with $N=N_{1}=N_{2}, w=$ $w_{1}=w_{2}$ respectively equal to $15,30,60$ and $5,10,20$.

\section{REFERENCES}

[1] L. Angeloni, D. Costarelli, G. Vinti, A characterization of the convergence in variation for the generalized sampling series, Ann. Acad. Sci. Fenn. Math., 43(2), (2018), 755-767.

[2] L. Angeloni, G. Vinti, Discrete operators of sampling type and approximation in $\varphi$-variation, Math. Nachr., 291(4), (2018), 546-555.

[3] C. Bardaro, P.L. Butzer, I. Mantellini, The exponential sampling theorem of signal analysis and the reproducing kernel formula in the Mellin transform setting, Sampling Theory in Signal and Image Process., 13(1), (2014), 35-66.

[4] C. Bardaro, P.L. Butzer, I. Mantellini, G. Schmeisser, On the Paley-Wiener theorem in the Mellin transform setting, J. Approx. Theory, 207, (2016), 60-75.

[5] C. Bardaro, P.L. Butzer, I. Mantellini, G. Schmeisser, A fresh approach to the Paley-Wiener theorem for Mellin transforms and the Mellin-Hardy spaces, Math. Nachr., 290, (2017), 2759-2774.

[6] C. Bardaro, P.L. Butzer, R.L. Stens, G. Vinti, Prediction by samples from the past with error estimates covering discontinuous signals, IEEE Trans. Information Theory, 56(1), (2010), 614-633.

[7] C. Bardaro, L. Faina, I. Mantellini, A generalization of the exponential sampling series and its approximation properties, Math. Slovaca, 67(6), (2017), 1481-1496.

[8] C. Bardaro, I. Mantellini, A note on the Voronovskaja theorem for Mellin-Fejer convolution operators, Appl. Math. Lett., 24, (2011), 2064-2067.

[9] C. Bardaro, I. Mantellini, Asymptotic behaviour of Mellin-Fejer convolution operators, East J. Approx., 17(2), (2011), 181-201.

[10] C. Bardaro, I. Mantellini, G. Schmeisser, Exponential sampling series: convergence in Mellin-Lebesgue spaces, Results Math., 74, (2019), Art. 119.

[11] M. Bertero, E.R. Pike, Exponential sampling method for Laplace and other dilationally invariant transforms I. Singularsystem analysis. II. Examples in photon correction spectroscopy and Frauenhofer diffraction, Inverse Problems, 7, (1991), $1-20 ; 21-41$. 
[12] A. Bobbio, M. Vassallo, G. Festa, Local Magnitude estimation for the Irpinia Seismic Network, Bull. Seismol. Soc. Am., 99, (2009), 2461-2470.

[13] P.L. Butzer, S. Jansche, A direct approach to the Mellin transform, J. Fourier Anal. Appl., 3, (1997), 325-375.

[14] P.L. Butzer, S. Jansche, The exponential sampling theorem of signal analysis, Atti Sem. Mat. Fis. Univ. Modena, Suppl. Vol. 46, (special issue dedicated to Professor Calogero Vint) (1998), 99-122.

[15] P.L. Butzer, R.L. Stens, Prediction of non-bandlimited signals in terms of splines of low degree, Math. Nachr., 132, (1987), $115-130$.

[16] P.L. Butzer, R.L. Stens, Linear prediction by samples from the past. In: R.J. Marks II (ed.) Advanced Topics in Shannon Sampling and Interpolation Theory,Springer, New York, (1993), 157-183.

[17] D. Casasent (Ed), Optical data processing, Springer, Berlin, (1978), 241-282.

[18] F. Gori, Sampling in optics In: R.J. Marks II (ed.), Advances Topics in Shannon Sampling and Interpolation Theory,Springer, New York, (1993), 37-83.

[19] B. Gutenberg, C. F. Richter, Discussion: Magnitude and energy of earthquakes, Science, 83, 2147, (1936), $183-185$.

[20] J.R. Higgins, Sampling theory in Fourier and signal analysis, Foundations, Oxford Univ. Press., Oxford, (1996).

[21] A. Kivinukk, G. Tamberg, On window methods in generalized Shannon sampling operators. In: G. Schmeisser and A. Zayed (Eds) New perspectives on approximation and sampling theory, 63-85, Appl. Numer. Harmon. Anal., Birkhaeuser Springer, Cham, (2014).

[22] R.G. Mamedov, The Mellin transform and approximation theory (in Russian), "Elm", Baku, (1991).

[23] N. Ostrowsky, D. Sornette, P. Parker, E.R. Pike, Exponential sampling method for light scattering polydispersity analysis, Opt. Acta, 28, (1994), 1059-1070.

[24] C. F. Richte, An instrumental earthquake magnitude scale, Bull. Seismol. Soc. Am., 25, (1935), 1-32.

[25] L.L. Schumaker, Spline functions: basic theory, John Wiley and Sons, New York, (1981).

[26] E. Weber, V. Convertito, G. Iannaccone, A. Zollo, A. Bobbio, L. Cantore, M. Corciulo, M. Di Crosta, L. Elia, C. Martino, A. Romeo, C. Satriano, An advanced seismic network in the southern Apennines (Italy) for seismicity investigations and experimentation with earthquake early warning Seism. Res. Lett., 78, (2007), 622-534.

[27] A.I. Zayed, Advances in Shannon's Sampling Theory, CRC Press, Boca Raton (1993).

\author{
UNIVERSITY OF PERUGIA \\ Department OF Mathematics AND COMPUTER SCIENCE \\ 1, Via VANVitelli, 06123 Perugia, Italy \\ Email address: carlo.bardaro@unipg.it \\ UNIVERSITY OF PERUGIA \\ Department OF MATHEMATiCS AND COMPUTER SCIENCE \\ 1, Via VANVITElli, 06123 Perugia, ItAly \\ Email address: giada.bevignani@gmail.com \\ UNIVERSITY OF PERUGIA \\ Department OF MATHEMATICS AND COMPUTER SCIENCE \\ 1, Via VANVITElli, 06123 Perugia, ItAly \\ Email address: ilaria.mantellini@unipg.it \\ UNIVERSITY OF PERUGIA \\ Department of Mathematics AND COMPUter SCIENCE \\ 1, Via VANVITElli, 06123 Perugia, Italy \\ Email address: marco.seracini@dmi.unipg.it
}

